\title{
OS EFEITOS DO EXERCÍCIO FÍSICO EM MEIO AQUÁTICO NO RISCO DE QUEDAS E EQUILÍBRIO EM UM GRUPO DE IDOSAS
}

\section{THE EFFECTS OF PHYSICAL EXERCISE IN AQUATIC ENVIRONMENT ON THE RISK OF FALLS AND BALANCE IN A GROUP OF OLDER WOMEN}

\author{
Angélica Lazzarotto Campagnolo', Jaqueline de Fátima Biazus², \\ Clándio Tim Marques ${ }^{3}$ e Alethéia Peters Bajotto ${ }^{4}$
}

\section{RESUMO}

Objetivos: Investigar os efeitos do exercício físico em meio aquático no que se refere a equilíbrio e risco de quedas de idosas participantes de grupo socioterápico. Metodologia: estudo de pesquisa quantitativo, retrospectivo e prospectivo, de intervenção quase experimental sem grupo controle. As participantes realizaram exercícios físicos em meio aquático por aproximadamente 21 meses, na frequência de duas vezes por semana. Foram avaliadas quanto ao risco de quedas (Timed up and go test) e equilíbrio (escala de Berg). Resultados: não houve uma diferença significativa nos valores do escore $B E R G$, pré e pós intervenção, $p=0,888$, mas encontrou-se diferença significativa nos valores do escore TUG, $p=0,035$. Conclusão: o exercício físico em meio aquático não apresentou influência sobre o equilíbrio de idosas que realizavam exercícios em grupo. Mas, quando comparados os resultados pré e pós-intervenção relacionados ao risco de quedas, houve uma diferença estatisticamente significativa, pois o risco de quedas diminuiu nesta amostra de idosas.

Palavras-chave: Hidroterapia; Qualidade de vida; Equilíbrio Postural; Assistência a Idosos.

\section{ABSTRACT}

Objectives: To investigate the effects of physical exercise in aquatic environment on the risk of falls and balance in older woman participants of a socio-therapic group. Methodology: quantitative, retrospective and prospective study of almost experimental intervention without control group. The participants performed physical exercises in an aquatic environment for approximately 21 months, twice a week. They were assessed for the risk of falls (Timed up and go test) and balance (Berg scale). Results: There was no significant difference

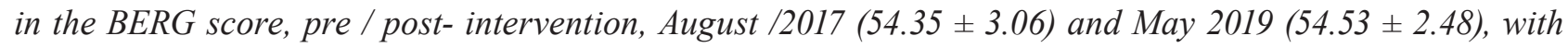
a value of $p=0.888$. However, a significant difference was found in the TUG score, August/2017 (13.29 \pm 4.26) and post-intervention, May 2019 (11.21 $\pm 2.29, p=0.035)$. Conclusion: physical exercise in the aquatic environment did not influenced the balance of the older women who performed exercises in group. However, when compared the pre-and post-intervention results related to the risk of falls, there was a statistically significant difference, where the risk of falls decreased in this sample of older women.

Keywords: Hidroteraphy; Quality of life; Postural Balance; Old Age Assistance.

\footnotetext{
${ }^{2}$ Fisioterapeuta, Graduada na Universidade Franciscana - UFN. E-mail: angelicacampagnolo2017@gmail.com

${ }^{3}$ Fisioterapeuta, Mestre, Docente do Curso de Fisioterapia - Universidade Franciscana - UFN. E-mail: jaquebiazus@ hotmail.com

${ }^{4}$ Doutor, Docente do Curso de Licenciatura em Matemática - Universidade Franciscana - UFN. E-mail: clandiomarques@ gmail.com

${ }^{5}$ Doutora, Docente do Curso de Fisioterapia - Universidade Franciscana - UFN. E-mail: aletheia@bajotto.com.br
} 
INTRODUÇÃO

Os números apontam que o envelhecimento no Brasil e no mundo aumentou significativamente nas últimas décadas. A população brasileira manteve a tendência de envelhecimento dos últimos anos e ganhou 4,8 milhões de idosos desde 2012, superando a marca dos 30,2 milhões em 2017. Os 4,8 milhões de novos idosos em cinco anos correspondem a um crescimento de $18 \%$ desse grupo etário, que tem se tornado cada vez mais representativo no Brasil. Estima-se que, em 2020, a população com mais de 60 anos no país chegue a 30 milhões de pessoas (13\% do total), e a expectativa de vida a 75,8 anos (ORGANIZAÇÃO MUNDIAL DA SAÚDE (OMS), 2005).

O processo conhecido como envelhecimento desencadeia alterações funcionais, morfológicas e bioquímicas, que vão modificando progressivamente o organismo, tornando-o mais propenso às agressões intrínsecas e extrínsecas que terminam por levá-lo à morte. Ao passar dos anos, com o avanço da idade, tornam-se comuns os comprometimentos, como a redução da força muscular, a diminuição da agilidade, da flexibilidade e da coordenação motora e perda do equilíbrio (ABDALA et al., 2017).

Dessa forma, estima-se que cerca de $30 \%$ dos idosos caem ao menos uma vez ao ano, o que torna o evento uma ocorrência relativamente comum aos idosos. A queda é o mais sério e frequente acidente doméstico que ocorre com idosos e a principal causa de morte acidental em pessoas acima de 65 anos. É responsável por 70\% das mortes acidentais em pessoas acima de 75 anos, podendo levar à diminuição da capacidade do idoso para realizar as atividades instrumentais da vida diária, causando um déficit para a independência, autonomia e qualidade de vida, além de representar importante morbidade, mortalidade, hospitalização, gastos com serviços sociais e de saúde (ABDALA et al., 2017).

Existe uma tendência que mulheres idosas apresentem maior propensão para quedas devido a menor massa magra e força muscular, maior prevalência de doenças crônico-degenerativas e exposição às atividades domésticas (BARTRÉS-FAZ; ARENAZA-URQUIJO, 2011). A prevenção de quedas perpassa o aprimoramento das condições de recepção de informações sensoriais do sistema vestibular, visual e somatossensorial de modo a ativar os músculos antigravitacionais para estimulação do equilíbrio. Uma das estratégias empregadas para a diminuição do risco de quedas é a prática da atividade física. A prática de atividades físicas e o envolvimento em atividades sociais são precursores que retardam o aparecimento de déficit na cognição (BREWSTER et al. 2014).

O exercício físico em meio aquático é um dos recursos que podem ser utilizados pela fisioterapia, uma vez que demonstra ser um facilitador para a realização de trabalho em grupos, pois promove a socialização, retarda e melhora as disfunções tanto físicas como psicológicas, comuns do envelhecimento (MEIRELES et al., 2010). Outros benefícios consequentes aos exercícios físicos em meio aquático são: promoção da funcionalidade, redução da dor, ganho de amplitude de movimento articular, aumento de força muscular, melhora do equilíbrio, melhora da autoestima e autoconfiança, 
prevenção de quedas, promoção da interação social e desenvolvimento de vínculos entre os participantes (SOUSA et al., 2016).

Portanto, sendo o envelhecimento populacional uma realidade vivenciada no Brasil e no mundo, e a queda para o idoso representando um preditor negativo para a saúde dessa população, é de extrema importância buscar estratégias que minimizem ou que evitem o risco de quedas. O exercício físico em meio aquático tem se mostrado capaz de aprimorar diversas variáveis que sofrem declínio no processo. Diante disso, este artigo justifica-se pela possibilidade de ir ao encontro de soluções que possam melhorar a saúde dessa população.

\section{MATERIAL E MÉTODOS}

Trata-se de uma pesquisa quantitativa, de intervenção quase experimental sem grupo controle, cujo objetivo foi avaliar a influência do exercício físico em meio aquático após 21 meses de intervenção, nas variáveis equilíbrio e risco de quedas de idosas. Este estudo foi aprovado pelo CEP/UFN sob o número de parecer 2.693.397. As participantes realizaram a atividade no Laboratório de Ensino e Pesquisa da Universidade Franciscana (LEP - UFN) da cidade de Santa Maria, de setembro de 2017 até junho de 2019, contando com aproximadamente 21 meses de duração e intervalos que correspondem a férias e recessos da Universidade. A intervenção fisioterapêutica em meio aquático ocorreu duas vezes na semana, com duração de 45 minutos cada sessão.

A população do estudo foi composta por idosas residentes na cidade de Santa Maria, RS, sendo do tipo não probabilística acidental. Foram incluídas idosas que tivessem 60 anos completos ou mais, que alcançassem pelo menos 23 pontos no Mini exame do estado mental (MEEM), estivessem aptas à realização de atividade física moderada a intensa, condição atestada por um médico (do último ano); capazes de deslocar-se até o Laboratório de Ensino e Pesquisa (LEP) da Universidade Franciscana e que estivessem residindo na cidade de Santa Maria.

As variáveis avaliadas por este protocolo de pesquisa foram o risco de quedas, por meio do teste Timed up and go test (TUGT), e o equilíbrio, por meio da Escala de Berg. O TUGT avalia o risco de quedas a partir de um teste funcional de mobilidade que serve para avaliar o equilíbrio sentado, transferência da posição de sedestação para bipedestação, estabilidade na deambulação e mudanças do curso da marcha sem usar estratégias compensatórias. Recomenda-se utilizar como valor de corte para risco de quedas 12,47 segundos, o qual parece ser o melhor preditivo nessa população, acima de 30 segundos para realização do teste, considera-se um grande risco de quedas para o idoso (ALEXANDRE et al., 2012). A escala de equilíbrio de BERG avalia o equilíbrio dinâmico e estático dos indivíduos e o risco de quedas, avalia o desempenho do equilíbrio funcional com 14 testes, sendo estes direcionados para a habilidade do indivíduo de sentar, ficar de pé, alcançar, girar em volta de si mesmo, olhar por cima de seus ombros, ficar em apoio unipodal e transpor degraus. Apresenta pontuação máxima de 56 pontos e 
mínima de 0 pontos, e cada teste possui cinco alternativas que variam de 0 a 4 pontos. As quedas são frequentes entre as pessoas com pontuações acima de 45 (ANSAI et al., 2014). Com base nos estudos de Alzayer, Beninato e Portney (2009), o ponto de corte da EEB foi analisado quanto a sensibilidade (S), especificidade (E), valor preditivo positivo (VPP) e negativo (VPN) do teste, razão de verossimilhança positiva (RVP) e negativa (RVN) para diagnóstico do risco de queda. Os pontos de corte utilizados para detectar o risco de queda foram: 45, 47, 49, 51 e 53.

As atividades iniciavam pelo acolhimento ao grupo, com aferição de pressão arterial e, em seguida, as participantes davam início ao protocolo de exercícios físicos aquáticos, divididos em: aquecimento, atividades de dupla tarefa, fortalecimentos gerais e alongamento, baseado no protocolo do estudo de Silva et al. (2019). O protocolo teve duração de 21 meses e intervalos que correspondem a férias e recessos da Universidade. A intervenção fisioterapêutica em meio aquático ocorreu duas vezes na semana, com duração de 45 minutos cada sessão.

Para os exercícios de dupla tarefa, eram executadas atividades em que a atenção das participantes é dividida entre uma atividade motora e cognitiva, concomitantemente. Um exemplo é a atividade de placas sinalizadoras. Acompanhada de música, essa atividade propõe que placas com cores e dimensões diferentes sejam associadas a tarefas, como: parar, deslocar- se à direita, deslocar-se à esquerda; seguir em frente; ir para trás, bater palmas acima da cabeça, por exemplo. Enquanto o grupo movia-se de acordo com o ritmo da música, os alunos começam a apresentar, um de cada vez, as placas, e as participantes deviam deslocar-se de acordo com o significado de cada placa, sem estímulo verbal.

\section{RESULTADOS}

O presente estudo contou com a participação de 17 idosas, com média de idade de 69,2 $\pm 8,1$ anos, em sua maioria casadas $(70,59 \%)$, de raça branca $(88,23 \%)$, aposentadas $(88,23 \%)$ e residentes de Santa Maria. Nenhuma idosa relatou histórico de queda durante o período em que participaram do grupo de exercícios físicos.

Foram analisados os resultados de BERG e TUG em agosto de 2017 (pré-intervenção) e maio de 2019 (pós-intervenção), inicialmente verificando-se a normalidade da amostra, utilizando-se o teste de Shapiro-Wilk. Em ambas as análises, foi aplicado o teste de Wilcoxon. Foi verificada a correlação entre as variáveis citadas e, para isso, utilizou-se a Correlação de Spearman, sendo os resultados analisados segundo Dancey e Reidy (2006), que apontam para uma classificação com $r=0,10$ até 0,39 (fraco); $r=0,40$ até 0,69 (moderado); $r=0,70$ até 1 (forte). Os resultados foram apresentados em forma de média \pm desvio-padrão. As diferenças foram consideradas significativas quando apresentaram o valor $-\mathrm{p}<0,05$. O software IBM SPSS Versão 25 foi utilizado como ferramenta computacional para a análise estatística dos dados. 
Comparando-se os valores pré e pós intervenção, percebe-se que não houve uma diferença significativa nos valores do escore BERG quando analisados em agosto de $2017(54,35 \pm 3,06)$ e maio de $2019(54,53 \pm 2,48)(\mathrm{p}=0,888)$.

Figura 1 - Valores individuais da escala BERG nos momentos pré (agosto de 2017) e pós-intervenção (maio de 2019).

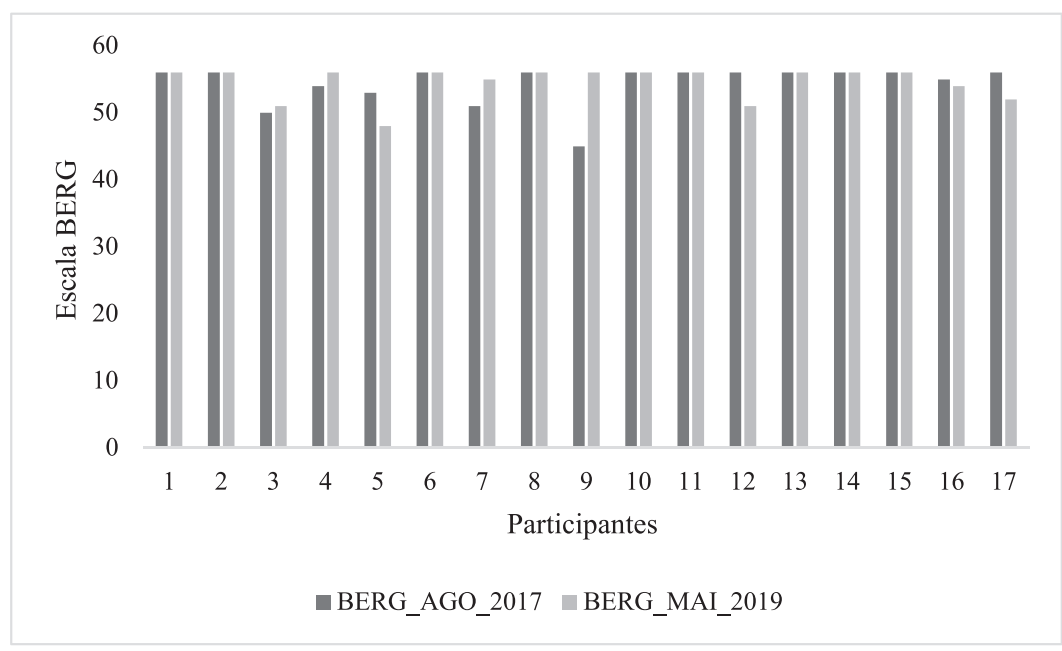

Fonte: elaboração própria.

\section{TIMED UP AND GO TEST (TUGT)}

Quando comparado o risco de quedas das idosas, encontrou-se diferença significativa nos valores do escore TUG quando comparados os resultados pré-intervenção - agosto de $2017(13,29 \pm 4,26)$ e pós-intervenção - maio de 2019 (11,21 \pm 2 ,29) ( $\mathrm{p}=0,035)$. Das 17 participantes, 12 (70,5\%) delas reduziram os valores e outras $5(29,5 \%)$ aumentaram, como mostrado no gráfico 2.

Figura 2 - Valores individuais do teste TUG nos momentos pré (agosto de 2017) e pós-intervenção (maio de 2019).

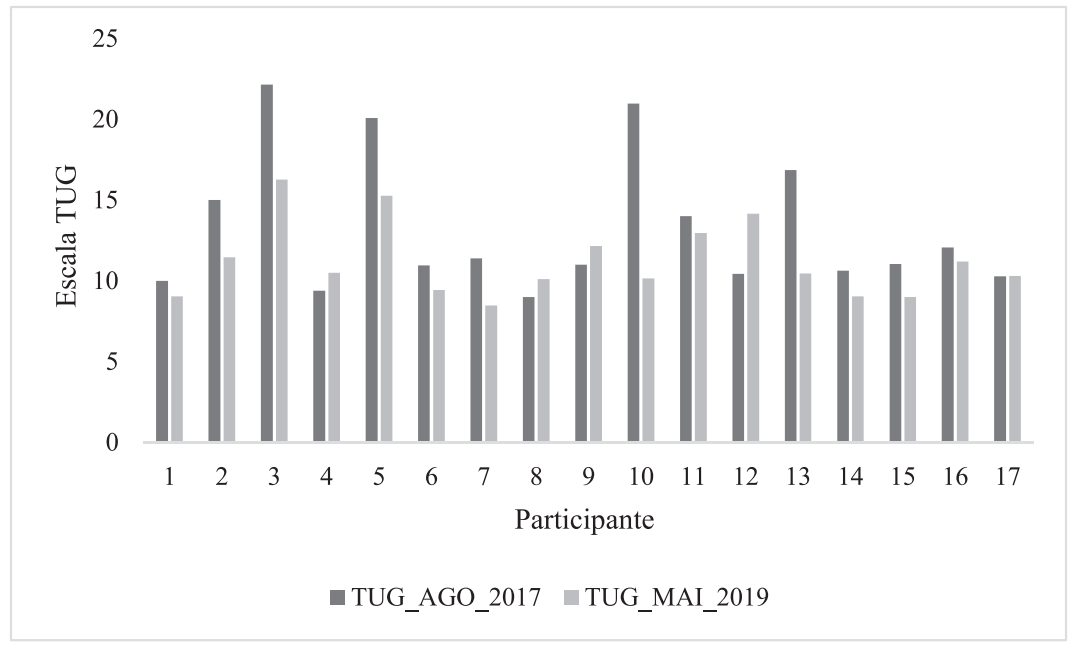

Fonte: elaboração própria. 


\section{DISCUSSÃO}

Neste estudo, teve-se como objetivo investigar os efeitos do exercício físico em meio aquático no risco de quedas e equilíbrio em um grupo de idosas que praticou exercício físico em grupo.

O envelhecimento é conceituado como um processo dinâmico e progressivo, em que ocorrem alterações funcionais, bioquímicas, morfológicas e psicológicas (ALCALDE et al., 2016). Sabe-se que o envelhecimento decorre de um conjunto de modificações funcionais e estruturais negativas do organismo que se acumulam de forma progressiva, por consequência do avanço da idade. Essas modificações, na maioria das vezes, prejudicam o desempenho de habilidades cognitivas e motoras, dificultando a adaptação do indivíduo ao meio ambiente, manifestando alterações de ordem psicológica e social.

Para a OMS, a queda é considerada um indicador de saúde e também um problema de saúde pública entre os idosos, principalmente os mais velhos. Conforme os dados da OMS, mundialmente, a taxa de quedas anual coloca-se entre 28 e 35\% na população com maior de 65 anos; a prevalência aumenta entre os idosos com mais de 70 anos para 32 e $42 \%{ }^{3}$.

Os exercícios aquáticos são uma estratégia chave para a população idosa, pois permitem que executem grandes movimentos, mas com menores riscos de quedas ou lesões, gerando aumento na força muscular, melhora na flexibilidade, no equilíbrio e, consequentemente, reduzindo o número de quedas (AVELAR et al., 2016).

Os resultados que a amostra estudada por este protocolo de pesquisa apresentou, após 21 meses de intervenção e aproximadamente 124 encontros, apontam para a manutenção do equilíbrio e diminuição do risco de quedas das idosas participantes do estudo. Isso significa que não houve diferença significativa para a escala BERG, ou seja, não houve melhora do equilíbrio. Ao analisar o comportamento do equilíbrio relacionado às 17 participantes, para 4, o equilíbrio piorou, para outras 4, o equilíbrio melhorou e para 9 participantes, o exercício físico manteve o escore de equilíbrio.

É necessário pontuar que a escala utilizada, embora validada e utilizada no mundo todo como referência em mensuração de equilíbrio em idosos, não foi sensível para a amostra, uma vez que as idosas que participaram das atividades apresentavam pouco déficit de equilíbrio. Esse comportamento foi percebido desde a primeira avaliação, tratando-se de uma amostra dotada de autonomia e boa capacidade de se locomover, permanecendo muito próxima da pontuação máxima da escala em todas as avaliações. Por esse motivo, em relação a essa amostra, é possível afirmar que o exercício realizado manteve o escore do equilíbrio para a maior parte da amostra.

Existe uma tendência à diminuição do equilíbrio, de instabilidade e quedas na população idosa. Equilíbrio envolve os estímulos sensoriais, o planejamento e a execução de movimentos para controlar a gravidade sobre a base de suporte, sendo realizado pelo sistema de controle postural (LEME; CARVALHO; SCHEICHER, 2017). 
Um estudo publicado por Siqueira et al. (2017), que teve como objetivo avaliar o efeito de um programa de fisioterapia aquática para o equilíbrio e a capacidade funcional de idosos, demonstrou que um programa de fisioterapia aquática pode melhorar o equilíbrio em idosos, minimizando os fatores de risco das quedas. Os resultados apresentaram melhora significativa para o grupo que realizou fisioterapia aquática tanto no equilíbrio postural como na marcha. A melhora no equilíbrio deve-se à atração molecular no meio líquido que, quando posta em movimento, gera uma resistência (viscosidade), que é responsável pelo suporte oferecido aos idosos durante a hidroterapia. Isso sugere melhora do equilíbrio dentro da água, o que reflete em melhora no solo.

Os grupos socioterápicos vêm se mostrando como uma estratégia para enfrentar fatores deletérios relativos ao processo de envelhecimento humano, incentivando nos idosos o resgate prazeroso de atividades sociais fora do ambiente social em que vivem, por meio da formação de um vínculo proporcionado pela interação entre os participantes, fazendo com que haja melhoria mental e física com a prática de atividades físicas (BAJOTTO; GOLDIM, 2011). Embora não seja um critério avaliado por este trabalho, acredita-se que a influência do grupo seja impactante na vida das idosas.

Quando avaliados os efeitos do exercício físico em meio aquático no risco de quedas das idosas participantes, encontrou-se diferença significativa após 21 meses de intervenção, em que a média do tempo de execução do teste diminuiu 2 segundos, da avaliação pré e pós-intervenção. Quando o teste é realizado em menos de 10 segundos, o participante apresenta baixo risco de quedas. A performance do teste em um intervalo de 10 a 20 segundos apresenta moderado risco de quedas, e é nesse intervalo que a amostra se encontra, e a média foi de 11,21 $\pm 2,29$ segundos. Quando o teste apresenta um tempo de execução acima de 20 segundos, representa alto risco de quedas. Cinco idosas aumentaram o tempo de realização do teste, porém nenhuma delas encontra-se em uma estratificação que denote grande risco de quedas.

O teste Timed up and Go apresenta originalmente como objetivo avaliar clinicamente alterações do equilíbrio dinâmico em idosos durante o desempenho de uma tarefa que predispõe à queda. Apesar de todas as características positivas do TUG, sua facilidade e baixo custo de execução, o tempo despendido para a realização é a sua única variável de desfecho, a qual não fornece informação sobre quais características poderiam estar associadas ao melhor ou pior desempenho (FARI et al., 2019). Dessa forma, o resultado deste protocolo de pesquisa aponta um risco moderado para a queda nessa amostra, porém não se pode atribuir o déficit a uma determinada característica.

Os achados de um estudo de Franciulli et al. (2015) mostraram que, após cinco semanas de intervenção, com exercícios realizados na piscina e no solo, os exercícios aquáticos foram mais efetivos na melhora dos indicadores de quedas. Segundo os autores, o grupo que realizou os exercícios aquáticos foi beneficiado pelos princípios físicos da água. Esses princípios, entre eles a viscosidade, o empuxo e a pressão hidrostática, permitem que o idoso diminua as compensações biomecânicas (base de suporte aumentada, diminuição da oscilação de membros superiores, 
aumento do ângulo de flexão dos joelhos, lordose cervical e cifose torácica), melhorando o controle postural, o equilíbrio e a marcha.

Segundo dados da Organização Mundial da Saúde (2005), a queda é considerada um indicador de saúde e também um problema de saúde pública entre os idosos, principalmente os mais velhos. Mundialmente, a taxa de quedas anual coloca-se entre 28 e $35 \%$ na população com maior de 65 anos; a prevalência aumenta entre os idosos com mais de 70 anos para 32 e $42 \%$.

Os exercícios aquáticos são uma boa estratégia para os idosos, pois permitem que eles executem movimentos maiores com menores riscos de queda ou lesão. A viscosidade da água permite que os movimentos sejam executados lentamente e, assim, os sujeitos têm mais tempo para criar e desenvolver mecanismos de reação de respostas. Os exercícios aquáticos resultam em aumento na força muscular, melhoram a flexibilidade, proporcionam maior equilíbrio e, consequentemente, reduzem o número de quedas (AVELAR et al., 2016).

O exercício de dupla tarefa, realizado durante as atividades em grupo, pode ser um fator que tenha auxiliado no resultado positivo em relação à diminuição de risco de quedas, pois o fator que ocasiona maior risco de quedas é justamente o mau desempenho na atividade de dupla tarefa. Essa afirmação não pode ser feita para esta amostra pois não houve um grupo controle para comparação dos resultados do grupo experimental, tratando-se de um viés deste estudo. A execução de dupla tarefa envolve a realização de uma principal - a tarefa simples, o principal centro de atenção - e uma tarefa secundária, desenvolvida simultaneamente, tornando-se, assim, uma atividade de dupla tarefa. Com o aumento da idade, acontece uma queda no desempenho da dupla tarefa, o que pode levar a uma perda no número de movimentos em um espaço de tempo específico, um aumento na quantidade de erros de execução, podendo chegar à não realização da tarefa (SILVA; DIAS; PIAZZA, 2017).

Segundo um estudo publicado por Silva, Dias e Piazza (2017), levando em consideração a importância de um bom desempenho em situações de dupla tarefa a fim de se manter uma boa mobilidade funcional e reduzir o risco de quedas durante a execução das atividades diárias, sugere-se que o profissional fisioterapeuta adicione, em seu programa de tratamento, trabalhos que envolvam situações de dupla tarefa, especialmente com os idosos residentes em instituições de longa permanência.

\section{CONCLUSÃO}

O exercício físico em meio aquático não apresentou influência sobre o equilíbrio de idosas que realizavam exercícios em grupo, após 21 meses de intervenção, mas, quando comparados os resultados pré-intervenção e pós-intervenção relacionados ao risco de quedas, houve uma diferença estatisticamente significativa, em que o risco de quedas - o tempo, em segundos - diminuiu nesta amostra de idosas. Estudos futuros poderão determinar, além de avaliar risco de queda e equilíbrio, 
qual déficit influencia diretamente no resultado. Além disso, um grupo controle poderia determinar o impacto que os exercícios físicos têm na vida dessas idosas.

\section{REFERÊNCIAS}

ABDALA, R. P. et al. Padrão de marcha, prevalência de quedas e medo de cair em idosas ativas e sedentárias. Revista Brasileira de Medicina do Esporte, v. 23, n. 1, p. 26-30, 2017.

ALEXANDRE, T. S. et al. Accuracy of Timed Up and Go Test for screening risk of falls among community-dwelling elderly. Revista Brasileira de Fisioterapia, v. 16, n. 5, p. 381-388, 2012.

ALZAYER, L.; BENINATO, M.; PORTNEY, L. G. The accuracy of individual Berg Balance Scale items compared with the total Berg score for classifying people with chronic stroke according to fall history. Journal of Neurologic Physical Therapy, v. 33, n. 3, 2009.

ANSAI, J. H. et al. Revisão de dois instrumentos clínicos de avaliação para predizer risco de quedas em idosos. Revista Brasileira de Geriatria e Gerontologia, v. 17, n. 1, p. 177-189, 2014.

ALCALDE, G. E. et al. Impacto do programa de fisioterapia aquática na mobilidade funcional de idosos da comunidade. Revista Kairós: Gerontologia, v. 19, p. 243-253, 2016.

BAJOTTO, A. P.; GOLDIM, J. R. Avaliação da qualidade de vida e tomada de decisão em idosos participantes de grupos socioterápicos da cidade de arroio do meio, RS, Brasil. Revista Brasileira de Geriatria e Gerontologia, v. 14, n. 4, p. 753-61, 2011.

BARTRÉS-FAZ, D.; ARENAZA-URQUIJO, E. M. B. T. Structural and functional imaging correlates of cognitive and brain reserve hypotheses in healthy and pathological aging. Brain Topography, v. 24, n. 3/4, p. 340, 2011.

BREWSTER, P. W. et al. Life experience and demographic influences on cognitive function in older adults. Neuropsychology, v. 28, n. 6, p. 846, 2014.

FARI, C. D. C. M. et al. TUG-ABS Português-Brasil. Revista Neurociências, v. 23, n. 3, p. 357-366, 2015.

FRANCIULLI, P. M. et al., Efetividade da hidroterapia e da cinesioterapia na reabilitação de idosos com histórico de quedas. Estudos interdisciplinares sobre o envelhecimento, v. 20, n. 3, 2015. 
AVELAR, I. S. et al. The influence of a protocol of aquatic exercises in postural control of obese elderly. Revista andaluza de medicina del deporte, 2016.

LEME, G.; CARVALHO, I.; SCHEICHER, M. Melhora do equilíbrio postural em mulheres idosas com o uso de informação sensorial adicional. Fisioterapia e Pesquisa, v. 24, n. 1, p. 68-73, 2017.

MEIRELES, A. E. et al. Alterações neurológicas fisiológicas ao envelhecimento afetam o sistema mantenedor do equilíbrio. Revista Neurociências, v. 18, n. 1, p. 103-108, 2010.

SILVA, R. J. M. da; DIAS, S. M. S.; PIAZZA, L. Desempenho em atividades de simples e dupla tarefas de idosos institucionalizados que realizam e não realizam fisioterapia. Fisioterapia e Pesquisa, v. 24, n.149-156, 2017.

SILVA, G. R. et al. Avaliação do torque muscular e sarcopenia em mulheres praticantes de fisioterapia aquática. Revista Kairós-Gerontologia, v. 22, n. 2, p. 555-568, 2019.

SIQUEIRA, A. F. et al. Efeito de um programa de fisioterapia aquática no equilíbrio e capacidade funcional de idosos. Revista Saúde e Pesquisa, v. 10, n. 2, p. 331-338, 2017.

SOUSA, L. M. M. et al. Risco de quedas em idosos residentes na comunidade: revisão sistemática da literatura. Revista Gaúcha de Enfermagem, v. 37, n. 4, 2016.

ORGANIZAÇÃO MUNDIAL DA SAÚDE (OMS). Envelhecimento ativo: uma política de saúde. 2005. 